\title{
ARTICLE \\ Perineuronal nets in the lateral hypothalamus area regulate cue-induced reinstatement of cocaine-seeking behavior
}

\author{
Jordan M. Blacktop ${ }^{1}$ and Barbara A. Sorg ${ }^{1}$
}

\begin{abstract}
We previously reported that a small, circumscribed region of the lateral hypothalamus, the anterior dorsal region (LHAad), stains heavily for PNNs and dense extracellular matrix (PNNs/ECM) with Wisteria floribunda agglutinin (WFA), and critically contributes to the acquisition of cocaine-induced conditioned place preference and cocaine self-administration. Here we tested the role of LHAad $\mathrm{PNNs} / \mathrm{ECM}$ in cue-induced reinstatement in cocaine self-administering (SA) rats and identified how it is embedded in the circuitry of motivated behavior and drug reward. Degradation of PNNs/ECM in the LHAad using chondroitinase $A B C$ (Ch-ABC) blocked the expression of cue-induced reinstatement of cocaine- but not sucrose-seeking behavior. We also identified for the first time the phenotype of LHAad PNN/ECM-surrounded neurons. LHAad neurons co-localized mainly with parvalbumin (PV+) and GABA. Predominant co-localization of WFA with VGLUT2 and GABA but not with GAD65/67 or glutamate indicates that the PNN/ECM-rich LHAad is predominantly GABAergic and receives dense glutamatergic input. The LHAad did not express significant amounts of melanin-concentrating hormone $(\mathrm{MCH})$, orexin, or galanin; neuropeptides that regulate both food-induced and cocaine-induced behavior. In addition, retrobead injections demonstrated that the LHAad receives robust prelimbic prefrontal cortex (PFC) input and provides moderate input to the prelimbic PFC and ventral tegmental area (VTA), with no apparent input to the nucleus accumbens. In summary, the dense PNN/ECM zone in the LHAad embedded within the circuitry associated with reward pinpoints a novel region that controls the expression of cocaine-seeking behavior.
\end{abstract}

Neuropsychopharmacology (2019) 44:850-858; https://doi.org/10.1038/s41386-018-0212-8

\section{INTRODUCTION}

Relapse is a significant obstacle to the long-term management of substance use disorders [1] which is hypothesized to be the result of long-term or permanent changes in the neurocircuitry of motivated behavior [2]. There still remains no effective FDAapproved pharmacotherapy for the treatment of cocaine addiction. Central to successful long-term cocaine relapse prevention is the need for further understanding of the circuitry of motivated behavior driving cocaine addiction.

The extracellular matrix (ECM) is an emerging contributor to cocaine-induced changes in the circuitry of motivated behavior in both humans [3] and rodents [4]. A specialized form of the ECM called perineuronal nets (PNNs) wrap around specific neurons, form during development, and appear to be involved in the etiology of addiction; referred hereafter as PNN/ECM. Degradation of the PNN/ECM can prevent cocaine-associated memory and the acquisition of cocaine-seeking behavior [5-7].

The medial forebrain bundle (MFB) is one of the most highly differentiated fiber systems in the circuitry of motivated behavior [8], and its activation is essential for reinforcement and motivation [9]. Prelimbic prefrontal cortex (PFC) and nucleus accumbens neurons travel via the MFB to provide input into the lateral hypothalamic area (LHA). The LHA, in turn, provides input into the ventral tegmental area (VTA) [8]. This pathway acts as a key integration site for motivation and reinforcement for both drug and non-drug reward [10], and its activation is necessary for relapse $[2,11]$.
The LHA is a highly heterogeneous brain region [8]. We recently defined a remarkably discrete region, the anterior dorsal LHA (LHAad), that expresses robust PNN/ECM. Degradation of LHAad PNN/ECM blocks the acquisition of cocaine- but not sucroseinduced conditioned place preference and self-administration (SA). No studies to our knowledge have defined the contribution of PNNs/ECM in the rat LHA in cue-induced reinstatement of cocaine seeking. Here we show that PNN/ECM removal after SA training blocks cue-induced reinstatement of cocaine but not sucrose seeking. We also show that the LHAad receives dense glutamatergic input and contains very low or undetectable levels of the feeding- and drug-related neuropeptides, orexin, $\mathrm{MCH}$, and galanin. We also describe for the first time that the LHAad is embedded within the neurocircuitry of motivated behavior by receiving heavy input from the prelimbic PFC while providing moderate output to the prelimbic PFC and VTA.

\section{MATERIALS AND METHODS}

Subjects

A total of 59 male Sprague-Dawley rats were used (16 cocaine SA; 15 sucrose SA; 17 retrobead injections: 6 VTA, 4 LHAad, 3 prelimbic PFC, 4 NA; 11 IHC: 3 galanin/orexin/MCH, 4 GABA, glutamate, CaMKIla, 4 VGLUT2/PV/GAD65/67). Rats weighing 300-330 g at the start of the experiment were singly housed and given food and water ad libitum except when placed in the SA apparatus. SA experiments were run during the dark cycle (lights off at 07:00 h).

\footnotetext{
${ }^{1}$ Department of Integrative Physiology and Neuroscience, Translational Addiction Research Center, Washington State University, Vancouver, WA 98686, USA
} Correspondence: Jordan M. Blacktop (jordan.blacktop@wsu.edu)

Received: 16 May 2018 Revised: 31 August 2018 Accepted: 2 September 2018

Published online: 11 September 2018 
All experiments were approved by IACUC according to the NIH Guide for the Care and Use of Laboratory Animals. All efforts were made to reduce animal suffering and to reduce the number of animals used.

\section{Drugs}

Cocaine hydrochloride (NIDA) was given by intravenous (IV; $0.5 \mathrm{mg} / \mathrm{kg} /$ infusion in $0.10 \mathrm{ml}$ over $6 \mathrm{~s}$ ) SA. Sucrose was dispensed for oral consumption ( $45 \mathrm{mg}$ pellets) SA. Chondroitinase-ABC (Ch$A B C)$, which degrades the PNN/ECM [12], was dissolved in sterile saline (vehicle) to a final concentration of $0.09 \mathrm{U} / \mu \mathrm{l}$, as previously reported [6].

\section{Surgery}

Rats were anesthetized with isoflurane followed by intramuscular zyket (ketamine $87 \mathrm{mg} / \mathrm{kg}$ + xylazine $13 \mathrm{mg} / \mathrm{kg}$ ). Only cocaine SA animals were implanted with a chronic indwelling IV catheter as previously reported. Catheter patency was maintained with $0.1 \mathrm{ml}$ of flushing solution (cefazolin $10 \mathrm{mg} / \mathrm{ml}, 0.1 \mathrm{ml}$ heparin $1000 \mathrm{U} / \mathrm{ml}$ ) to prevent infection and catheter occlusion.

Bilateral cannula/obturators $1 \mathrm{~mm}$ above the LHAad were implanted using the following coordinates: $0^{\circ}$ angle away from midline; $\mathrm{A} / \mathrm{P}-1.8 \mathrm{~mm}$ from bregma; $M / L \pm 1.3 \mathrm{~mm}$ from midline; $D / V-6.4 \mathrm{~mm}$ from skull surface. Rats recovered for 7 days before the start of the experiment. Microinjection needles, $1 \mathrm{~mm}$ longer than cannula were used to deliver a volume of $0.6 \mu \mathrm{l}$ of the enzyme Ch-ABC or vehicle bilaterally over a period of $108 \mathrm{sec}$, as previously reported $[2,6]$.

In separate naïve rats, retrobead injections were conducted using a modified protocol according to Schofield [13]. Rats underwent unilateral infusions of the Rhodamine-conjugated microspheres retrograde tracer (RetroBeadsTM Lumafluor, USA; one right unilateral $0.25 \mu \mathrm{l}$ injection per animal). Rats are anesthetized with brief exposure to isoflurane followed by an intramuscular injection of zyket (ketamine $87 \mathrm{mg} / \mathrm{kg}+$ xylazine $13 \mathrm{mg} / \mathrm{kg}$ ) and placed into the stereotaxic apparatus. A KH (knurled hub) needle from a Hamilton 7000 series $1 \mu$ l syringe was used as the microinjector and secured onto the stereotaxic arm which was attached to a $1 \mu \mathrm{l}$ syringe placed within an injection pump with PE 20 polyethylene tubing. A single burr hole ( $2 \mathrm{~mm}$ in diameter) was made by first thinning the skull and then using a bent small needle $(27 \mathrm{G})$ to perform a craniotomy, perforating the skull and dura with the tip of the needle. The tip of the microinjector was placed into a non-diluted retrobead stock aliquot, manually withdrawn using the Hamilton plunger within the microinfusion pump, slowly, to a final volume of $0.5 \mu \mathrm{l}$. Mineral oil was applied to the tip of the microinjector to prevent clogging then lowered into the injection site at a rate of $1 \mathrm{~mm} / \mathrm{min}$ using the following coordinates: LHAad, $0^{\circ}$ angle away from midline, $A / P$ $-1.8 \pm 1.3 \mathrm{~mm}$ from midline, $D / V-7.4 \mathrm{~mm}$ from skull surface; VTA, $12^{\circ}$ angle away from midline, $A / P-5.6 \pm 2.5 \mathrm{~mm}$ from midline, $D / V$ $-7.7 \mathrm{~mm}$ from skull surface; prelimbic PFC, $0^{\circ}$ angle away from midline, $A / P+3.7 \pm 0.8 \mathrm{~mm}$ midline, $D / V-4.0 \mathrm{~mm}$ from skull surface; nucleus accumbens, $0^{\circ}$ angle away from midline, $A / P+$ $1.4 \pm 1.2 \mathrm{~mm}$ from midline, $D / V-7.2 \mathrm{~mm}$ from skull surface. Using an automated infusion pump, $0.25 \mu \mathrm{l}$ was pressure injected at a rate of $0.1 \mu \mathrm{l} / \mathrm{min}$. Iontophoretic application of retrobeads is not recommended [13]. The microinjector remained in place for an additional $10 \mathrm{~min}$ to allow for full diffusion then removed at a rate of $1 \mathrm{~mm} / \mathrm{min}$. Rats were euthanized 10 days following injection.

\section{Self-administration}

SA chambers were equipped with active and inactive levers, cue lights over each lever, and a house light. Reinforced lever presses resulted in reward delivery, cue light activation, and a $20 \mathrm{~s}$ timeout period. Active and inactive lever presses during the time out period resulted in no consequences but were recorded. SA consisted of $2 \mathrm{~h}$ sessions, 5 days a week, at a fixed-ratio of 1 (FR1), for a total of 7-10 days to self-administer cocaine or sucrose. During extinction, reward and cue-light were both omitted. Upon reaching criterion $(<20$ total active lever presses; 10 days for cocaine and 5 days for sucrose $S A$ ) Ch-ABC or vehicle was administered into the LHAad approximately $16 \mathrm{~h}$ before a single $30 \mathrm{~min}$ reinstatement session that was started with a single cue light prime. Rats were sacrificed $2 \mathrm{~h}$ following testing, brains were removed, and placement (cannula and PNN degradation) was verified.

\section{Immunohistochemistry}

Free-floating $30 \mu \mathrm{m}$ sections were washed in PBS, placed in $50 \%$ ethanol for $30 \mathrm{~min}$, washed in PBS, placed in $3 \%$ blocking serum for $1 \mathrm{~h}$, and then incubated overnight at $4{ }^{\circ} \mathrm{C}$ with primary antibodies. The primary antibodies used were against parvalbumin, CaMKIla, VGLUT2, GAD65/67, GABA, glutamate, galanin, orexin, and $\mathrm{MCH}$ (refer to Table $\mathrm{S} 1$ ). The following day, sections were washed in PBS, incubated for $2 \mathrm{~h}$ with secondary antibody (AlexaFluor594; 1:200), incubated for $2 \mathrm{~h}$ at room temperature with fluorescein-labeled Wisteria floribunda agglutinin (WFA; 1:500), washed in PBS, mounted onto slides, coverslipped, and imaged using a Leica laser scanning confocal microscope. WFA histochemistry binds to the chondroitin sulfate side chains, major components in both the ECM, and the more specialized ECM form the PNN [14].

\section{Cell counting}

Retrobead quantification was performed using the automated analysis macro Perineuronal net Intensity Program for the Standardization and Quantification of ECM Analysis (PIPSQUEAK) for use with FIJI/ImageJ software [15].

\section{Statistics}

Statistical tests were conducted using Prism 6 and Statistica. A two-way repeated measures analysis of variance (ANOVA) was conducted on behavioral data (vehicle vs. Ch-ABC treatment as the between-subjects measure and SA day as the within-subjects measure) followed with Neuman-Keuls post-hoc test in the case of a significant interaction. Further main effects were conducted using an unpaired Student's two-tailed $t$-test. Differences were considered significant when $p<0.05$.

\section{RESULTS}

LHAad PNN/ECM degradation

The LHAad is characterized by robust PNN/ECM expression using the PNN marker WFA (Fig. 1b), is found dorsolateral to the fornix and ventromedial to the internal capsule (Fig. 3a), and can be abolished by Ch-ABC administration (Fig. 1C). Injection sites of cocaine (Fig. 1k) and sucrose (Fig. 2i) trained animals that received intra-LHAad microinjection of vehicle (black circles) and Ch-ABC (gray circles) were considered a successful microinjection site when $\mathrm{Ch}-\mathrm{ABC}$ degraded a significant $(>50 \%)$ proportion PNN/ECM expression in the LHAad. Three SA rats and three RB rats were omitted from the analysis for placement outside the area of interest.

LHAad PNN/ECM degradation blocks cue-induced reinstatement of cocaine seeking

To test whether the LHAad PNN/ECM was necessary for expression of cue-induced reinstatement of cocaine seeking, a separate cohort of rats was treated with either intra-LHAad vehicle or Ch$A B C$ the day prior to a single cue-induced reinstatement session. Figure 1a shows the timeline of the cocaine SA experiments. There were no differences between rats treated with vehicle or $\mathrm{Ch}-\mathrm{ABC}$ (Table S2) on the total active lever presses (vehicle $335 \pm 100, n=$ 7; Ch-ABC $265 \pm 71 n=9 ; p=0.57$ ), total reinforced lever presses (vehicle 188 \pm 142 ; Ch-ABC $162 \pm 25 ; p=0.59$ ), or total inactive 
a



b

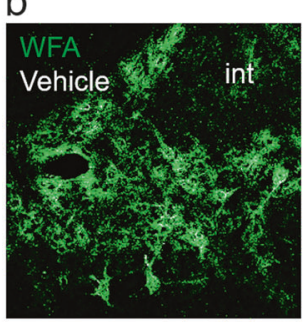

C

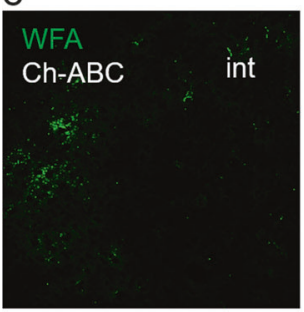

d

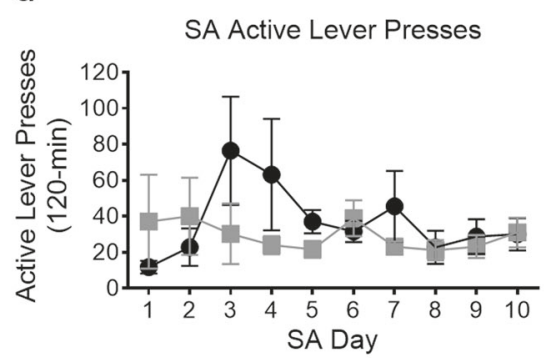

g

屯

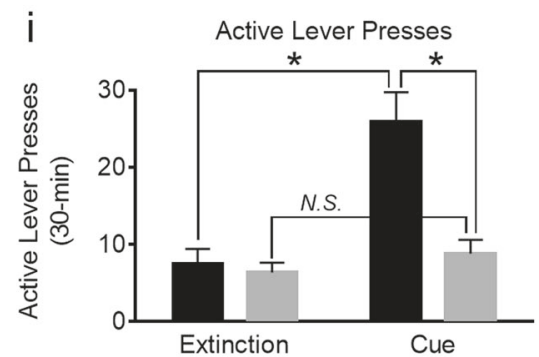

e

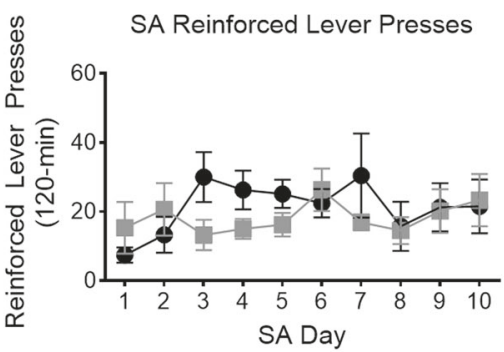

Vehicle $\mathrm{n}=7$

Ch-ABC $n=9$

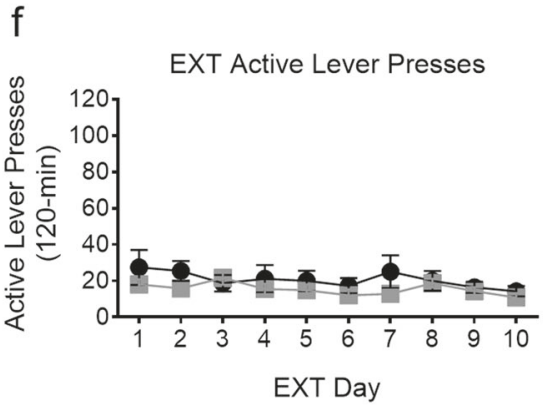

h
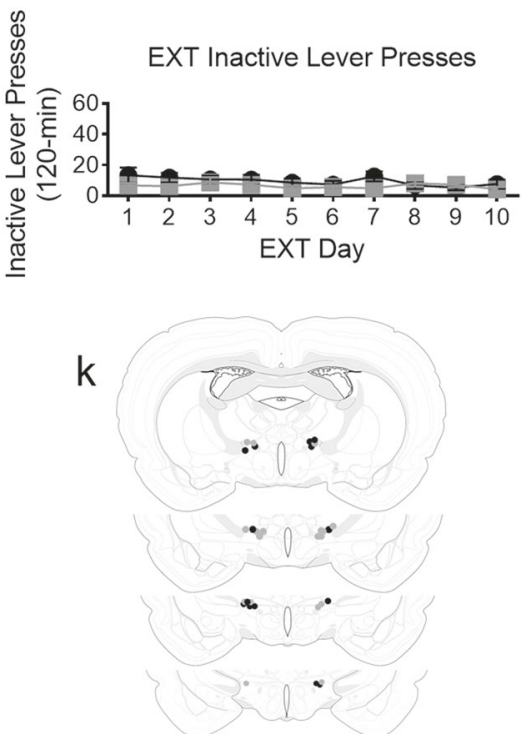

Fig. 1 LHAad PNN/ECM degradation blocks cue-induced reinstatement of cocaine seeking. a Timeline of the experiment. $\mathbf{b}$ Depiction of the targeted region characterized by robust PNN/ECM expression using WFA. This area is the lateral hypothalamic area (LHA), anterior region (LHAa), dorsal (LHAd) zone (LHAad) ventromedial to the internal capsule (int). c Strong PNN/ECM expression in the LHAad is abolished by ChABC administration. d SA active lever presses, e SA reinforced lever presses, and $\mathbf{f}$ EXT active lever presses. g SA inactive lever presses, and h EXT inactive lever presses. $\mathbf{i}$ Active lever presses in the first 30-min of the last extinction session compared to the 30 min cue-induced reinstatement session, and $\mathbf{j}$ inactive lever presses during the $30 \mathrm{~min}$ cue-induced reinstatement session (Data are mean \pm SEM). Differences were considered significant when ${ }^{*} p<0.05$. $\mathbf{k}$ Injection sites in cocaine trained animals that received intra-LHAad microinjection of vehicle (black circles; $n=7$ ) and Ch-ABC (gray circles; $n=9$ )

lever presses (vehicle $59 \pm 14$, Ch-ABC $105 \pm 24, p=0.16$ ) during SA.

For self-administration training, a two-way repeated measures ANOVA of active lever presses (Fig. 1d) showed a main effect of day $\left(F_{9,72}=2.418, p=0.0185\right)$, no effect of drug treatment $\left(F_{1,8}=\right.$ $1.371, p=0.275)$, and day $\times$ treatment interaction $\left(F_{9,72}=2.940\right.$, $p=0.005$ ). Neuman-Keuls post-hoc analysis reported significance only on day 3; treatment groups were not different on any other SA day. Reinforced lever presses (Fig. 1e) did not show a main effect of day $\left(F_{9,72}=1.575, p=0.1392\right)$, drug treatment $\left(F_{1,8}=\right.$ $0.26257, p=0.622)$, or a day $\times$ treatment interaction $\left(F_{9,72}=1.389\right.$, $p=0.209$ ). Inactive lever presses (Fig. $1 \mathrm{~g}$ ) did not show a main effect of day $\left(F_{9,72}=1.927, p=0.0615\right)$, treatment $\left(F_{1,8}=0.197\right.$, $p=0.669)$, or a day $\times$ treatment interaction $\left(F_{9,72}=1.771, p=\right.$ 0.0889 ). For extinction training, a two-way repeated measures ANOVA of active lever presses (Fig. 1f) did not show a main effect of day $\left(F_{9,126}=1.77, p=0.08\right)$, treatment $\left(F_{1,14}=1.29, p=0.275\right)$, or a day $\times$ treatment interaction $\left(F_{9,126}=0.934, p=0.494\right)$. Inactive lever presses (Fig. $1 \mathrm{~h})$ did not show a main effect of day $\left(F_{9,126}=\right.$ $1.897, p=.058)$ or treatment $\left(F_{1,14}=1.512, p=0.239\right)$, but did report a day $\times$ treatment interaction $\left(F_{9,126}=1.989, p=0.0457\right)$.

To test whether the LHAad PNN/ECM is involved in cue-induced reinstatement of cocaine $S A$, we administered intra-LHAad Ch-ABC prior to a single $30 \mathrm{~min}$ reinstatement session. The effects of vehicle and $C h-A B C$ injection into the LHAad on the cue-induced reinstatement of total active lever presses during the first $30 \mathrm{~min}$ 


\begin{tabular}{|c|c|c|}
\multicolumn{3}{|c|}{ Veh or Ch-ABC } \\
\hline $7-10$ day & $5 \mathrm{~d}$ & 30 min cue- \\
\hline sucrose $(2 \mathrm{hr}) \mathrm{SA}$ & Extinction & reinstate
\end{tabular}
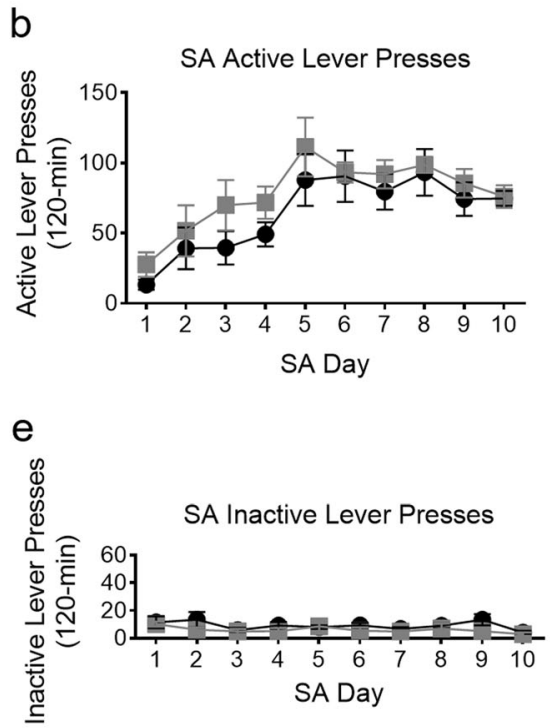

g

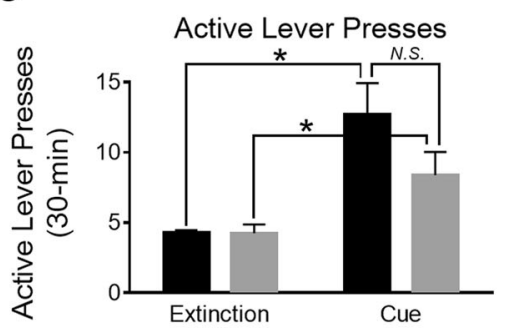

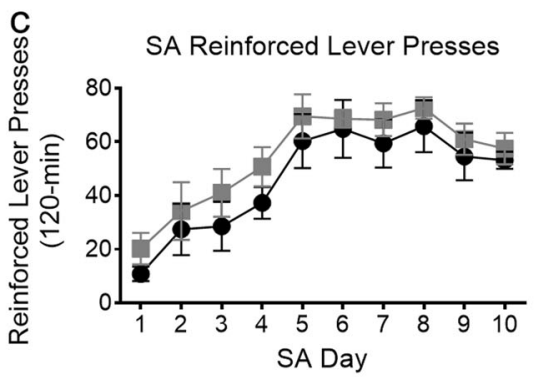
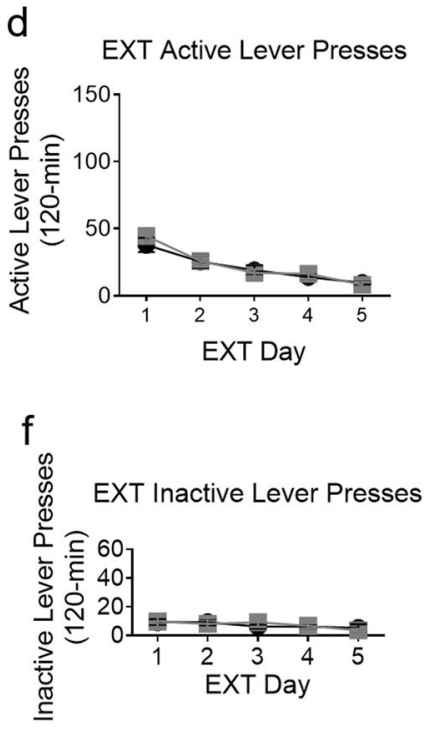

h

Vehicle $n=7$

Ch-ABC $n=8$

Cue

Inactive Lever Presses
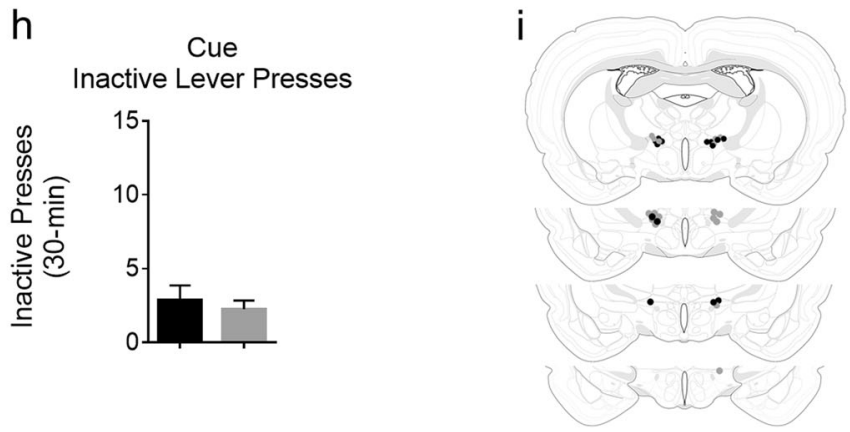

Fig. 2 LHAad PNN/ECM degradation does not block cue-induced reinstatement of sucrose seeking. $\mathbf{a}$ Timeline of the experiment. $\mathbf{b}$ SA active lever presses, c SA reinforced lever presses, d EXT active lever presses, e SA inactive lever presses, and f EXT inactive lever presses. $\mathbf{g}$ Active lever presses in the first $30 \mathrm{~min}$ of the last extinction session compared to the 30 min cue-induced reinstatement session, and $\mathbf{h}$ inactive lever presses during the 30 min cue-induced reinstatement session (Data are mean \pm SEM). Differences were considered significant when ${ }^{*} p<0.05$. i Injection sites in sucrose trained animals that received intra-LHAad microinjection of vehicle (black circles; $n=7$ ) and Ch-ABC (gray circles; $n=8)$

of the last extinction session (for comparison) and during the cueinduced reinstatement session are shown in Fig. 1i. A two-way repeated measures ANOVA of active lever presses revealed a main effect of day $\left(F_{1,14}=38.74, p<0.0001\right)$, treatment $\left(F_{1,14}=10.79\right.$, $p=0.0054)$, and an interaction $\left(F_{1,14}=22.72, \quad p=0.0003\right)$. Neuman-Keuls post-hoc analysis demonstrated active lever presses were different between treatment groups on the cuereinstatement day. Active lever presses $\left(F_{1,14}=3.56, p=0.0007\right)$ and inactive lever presses (Fig. 1 ; $; F_{1,14}=3.542, p=0.234$ ) were lower in the Ch-ABC treatment group. For active lever presses, only vehicle-treated rats showed cue-induced reinstatement compared with their last extinction day $\left(F_{1,12}=3.831\right.$, $p=0.0012)$, while $C h-A B C$-treated rats did not $\left(F_{1,16}=2.048, p=\right.$ 0.2863). These data indicate that $C h-A B C$ treatment blocked cueinduced reinstatement of cocaine-seeking behavior.

LHAad PNN/ECM degradation does not block cue-induced reinstatement of sucrose seeking

To test whether the LHAad PNN/ECM was necessary for expression of cue-induced reinstatement of sucrose seeking, a separate cohort of rats was treated with either vehicle or Ch-ABC administration into the LHAad prior to a single cue-induced reinstatement session. Figure $2 \mathrm{a}$ shows the timeline of the sucrose SA experiments. There were no differences between rats treated with vehicle or Ch-ABC (Table S2) on the number of total active lever presses (vehicle $641 \pm 76, n=7$; Ch-ABC $778 \pm 55 n=8 ; p=$ $0.16)$, correct active lever presses (vehicle $462 \pm 55$, Ch-ABC $544 \pm$ $36, p=0.23$ ), or total inactive lever presses (vehicle $90 \pm 18$, Ch$\mathrm{ABC} 60 \pm 17, p=0.23$ ) during SA.

For self-administration training, a two-way repeated measures ANOVA of active lever presses (Fig. 2b) showed a main effect of day $\left(F_{9,117}=9.032, p<0.0001\right)$, no effect of drug treatment $\left(F_{1,13}=2.199, p=0.1620\right)$, and no day $\times$ treatment interaction $\left(F_{9,117}=0.3149, p=0.9687\right)$. Reinforced lever presses (Fig. $2 c$ ) showed a main effect of day $\left(F_{9,117}=15.93, p<0.0001\right)$, but no effect of treatment $\left(F_{1,13}=1.608, p=0.2270\right)$ or of a day $\times$ treatment interaction $\left(F_{9,117}^{\prime}=0.1204, p=0.9991\right)$. Inactive lever presses (Fig. 2e) showed a main effect of day $\left(F_{9,117}=2.375, p=\right.$ $0.0167)$ but no effect of treatment $\left(F_{1,13}=1.568, p=0.2325\right)$, or of a day $\times$ treatment interaction $\left(F_{9,117}=0.1005, p=0.4399\right)$. For 
a

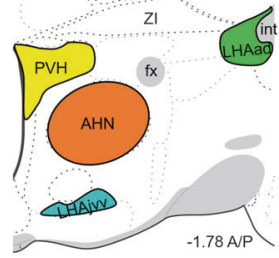

C

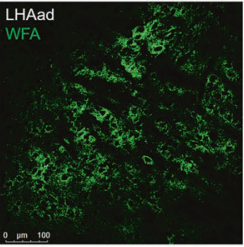

d

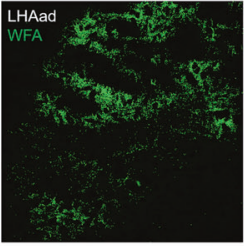

e
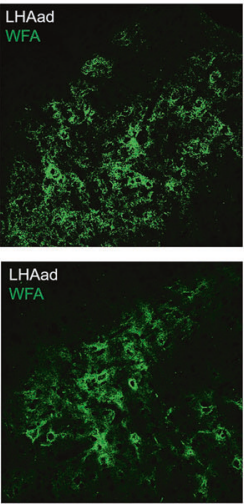

g

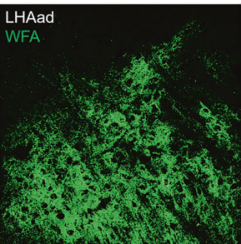

h

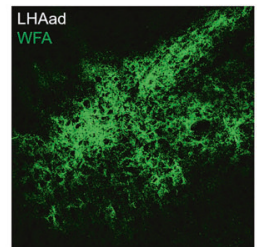

b
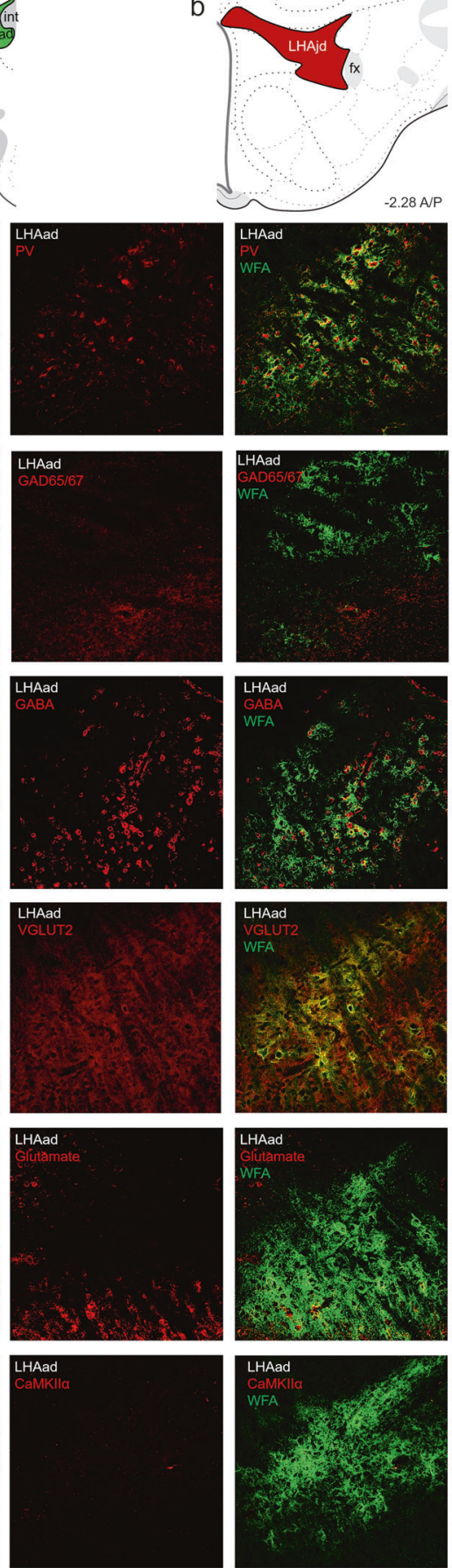

i

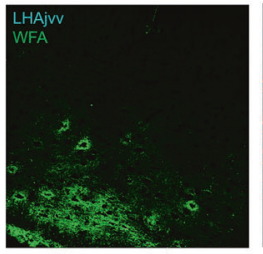

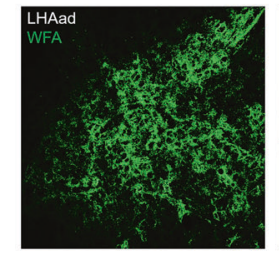

$\mathrm{k}$

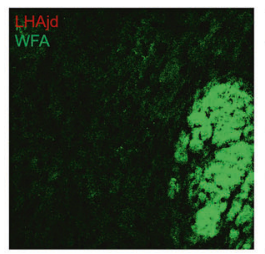

I

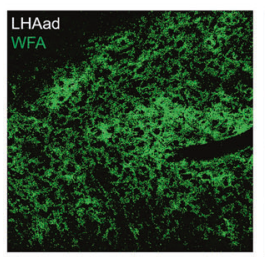

$\mathrm{m}$

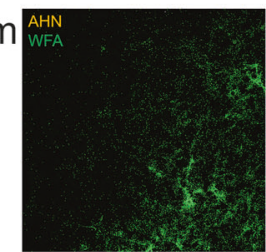

$\mathrm{n}$

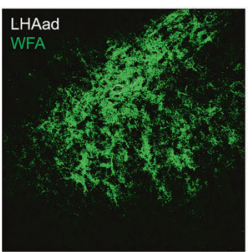

$\mathrm{O}$

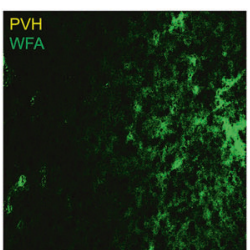

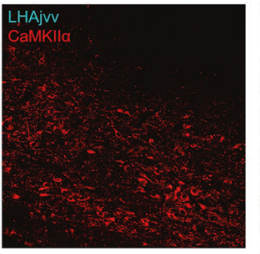
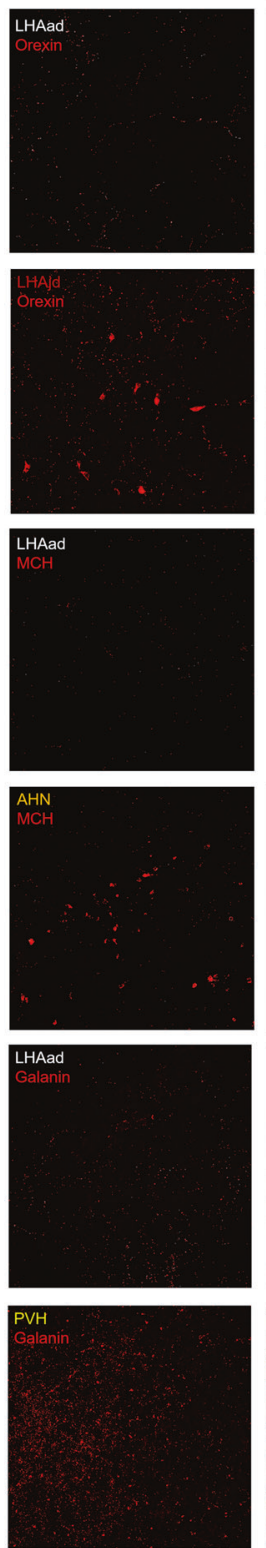
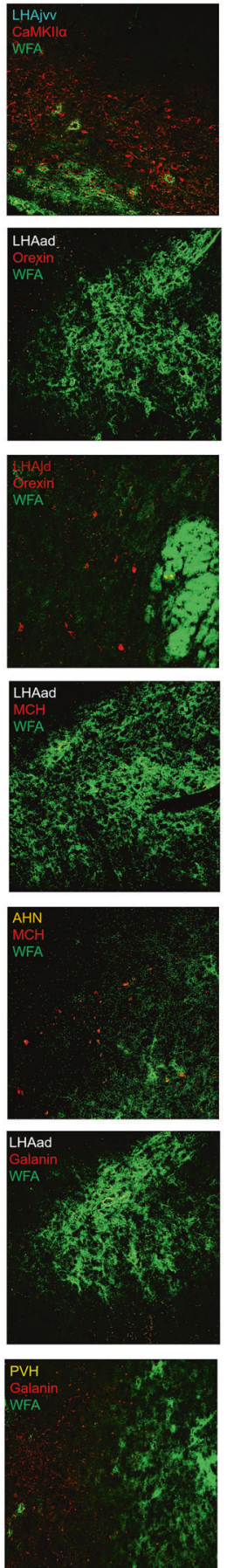

Fig. 3 LHAad neurons are GABAergic $\mathrm{PV}^{+}$neurons, receive dense glutamatergic input, and do not co-localize with neuropeptides involved in feeding. Positive control regions were the a anterior hypothalamic nucleus (AHN), ventral juxtaventromedial LHA (LHAjvv), paraventricular hypothalamus (PVH), and b juxtadorsomedial LHA (LHAjd). c WFA colabels with parvalbumin (PV), d WFA does not colabel with GAD65/67, e WFA colabels with GABA, $\mathbf{f}$ WFA colabels with VGLUT2, $\mathbf{g}$ WFA only colabels with glutamate near the ventral tier of the LHAad, $\mathbf{h}$ WFA does not colabel with CaMKIl $\alpha$, i LHAjvv CaMKIl $\alpha$ positive control, j minimal orexin expression in the LHAad, $\mathbf{k}$ LHAjd orexin positive control, I minimal $\mathrm{MCH}$ expression in the LHAad, $\mathbf{m}$ AHN MCH positive control, $\mathbf{n}$ minimal galanin expression in the LHAad, $\mathbf{o}$ PVH galanin positive control ( $n=11$ total: 3 galanin/orexin/MCH; 4 GABA, glutamate, CaMKIl $\alpha ; 4$ VGLUT2/PV/GAD65/67)

extinction training, a two-way repeated measures ANOVA of active lever presses (Fig. 2 d) showed a main effect of day $\left(F_{4,52}=41.21\right.$, $p<0001)$ but no effect of treatment $\left(F_{1,13}=0.089, p=0.7706\right)$, or of a day $\times$ treatment interaction $\left(F_{4,52}^{\prime}=0.8230, p=0.5165\right)$. Inactive lever presses (Fig. 2f) reported a main effect of day $\left(F_{4,52}=2.879, p=.0315\right)$ but no effect of treatment $\left(F_{1,13}=0.0049\right.$, $p=0.9455)$, or of a day $\times$ treatment interaction $\left(F_{4,52}=0.5975\right.$, $p=0.6661)$.

To test whether the LHAad PNN/ECM is involved in cue-induced reinstatement of sucrose SA, intra-LHAad Ch-ABC was administered prior to a single $30 \mathrm{~min}$ reinstatement session. The effects of vehicle and $C h-A B C$ injection into the LHAad on the cue-induced reinstatement of active lever presses during the first $30 \mathrm{~min}$ of the 
PL to LH

a

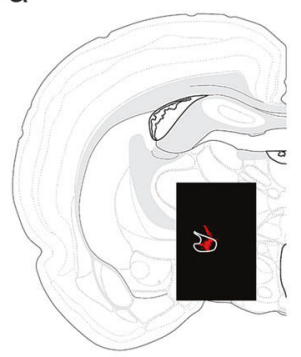

LH to VTA

d

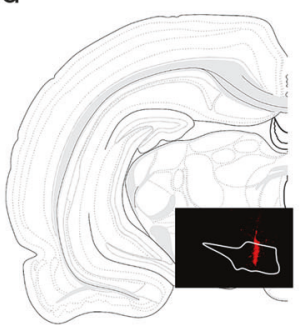

\section{LH to PL}

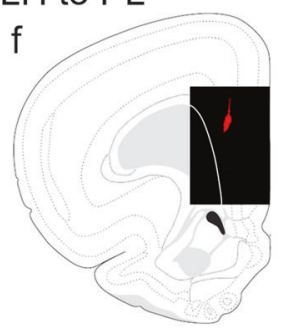

g
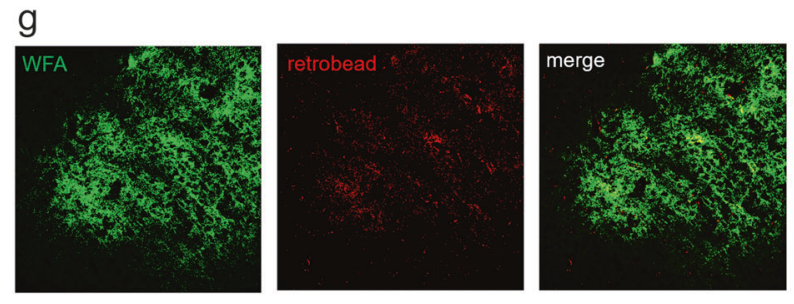

\section{LH to NA}

h

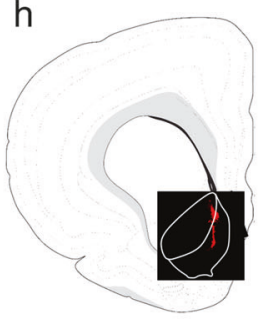

j
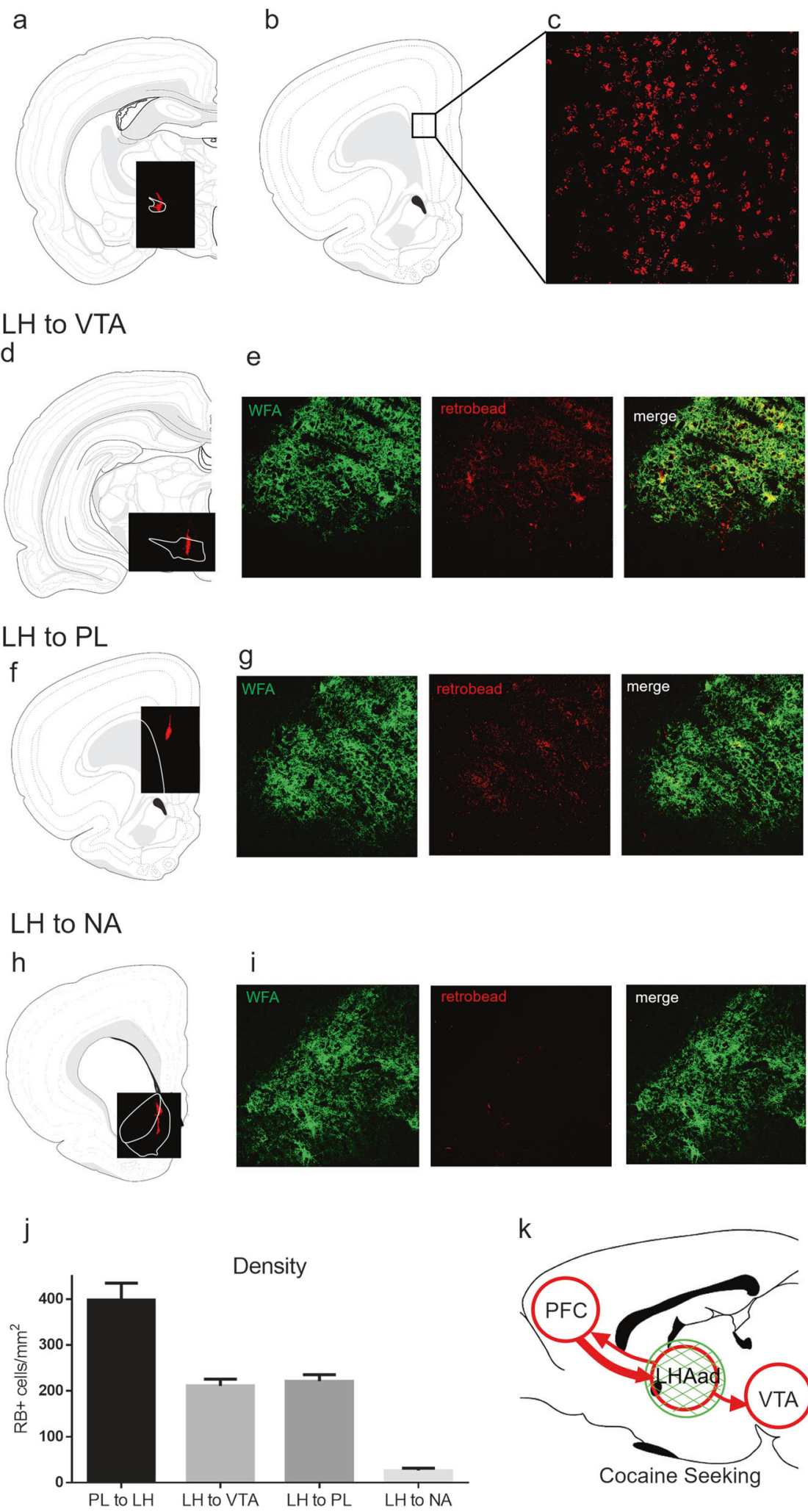

e
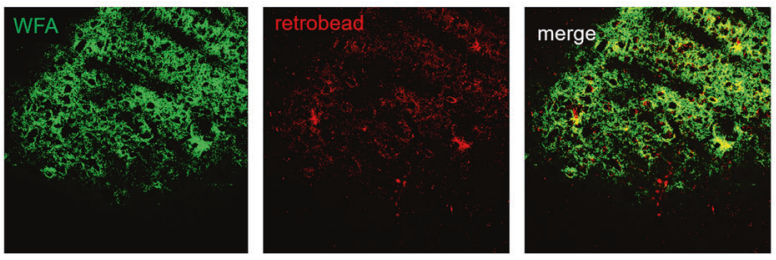

i
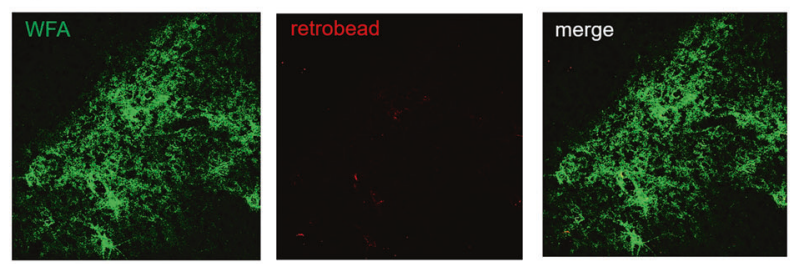

k

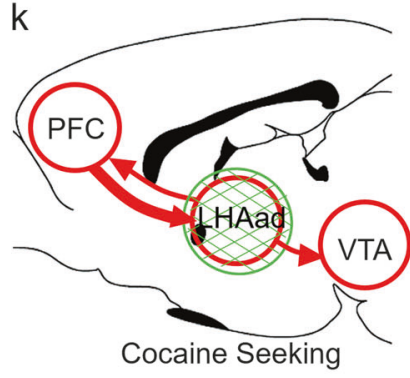

Fig. 4 Neurocircuitry of the LHAad using retrobead injections. a Injections into the LHAad resulted in dense retrobead expression in the prelimbic PFC (b, c). Injections into the VTA (d) resulted in moderate expression in the LHAad (e). Injections into the (f) prelimbic PFC resulted in moderate expression in the LHAad $(\mathbf{g})$. Injections into the $\mathbf{h}$ nucleus accumbens $\mathbf{i}$ resulted in minimal expression in the LHAad. j Quantification of the density of RB + cells $/ \mathrm{mm}^{2}$. k Summary of LHAad neurocircuitry receiving robust prelimbic PFC (thicker arrow) inputs, while providing moderate outputs (thin arrows) to the prelimbic PFC and VTA ( $N=17$ total: 6 VTA; 4 LHAad; 3 prelimbic PFC; 4 NA) 
856

last extinction session (for comparison) and during the cueinduced reinstatement session are shown in Fig. $2 \mathrm{~g}$. A two-way repeated measures ANOVA of active lever presses revealed a main effect of day $\left(F_{1,13}=25.62, p=0.0002\right)$, but no effect of treatment $\left(F_{1,13}=2.35, p=0.1994\right)$, or of a day $\times$ treatment interaction $\left(F_{1,13}\right.$ $=3.010, p=0.1064)$. Active lever presses $\left(F_{1,13}=1.558\right.$, $p=0.1348$ ) and inactive lever presses (Fig. 2h; $F_{1,13}=2.564$, $p=0.6013$ ) were not different between groups. For active lever presses, both vehicle $\left(F_{1,12}=143.8, p=0.0025\right)$ and Ch-ABC $\left(F_{1,14}\right.$ $=7.157, p=0.0352$ ) treated rats demonstrated cue-induced reinstatement compared with the last extinction day. These data indicate that $\mathrm{Ch}-\mathrm{ABC}$ treatment did not block cue-induced reinstatement of sucrose-seeking behavior.

LHAad neurons are GABAergic $\mathrm{PV}^{+}$neurons, receive dense glutamatergic input, and do not co-localize with neuropeptides involved in both food and drug reward

Different nuclei of the hypothalamus were targeted as positive controls for immunofluorescence of several different markers when there was absence of their expression in the LHAad (Fig. 3a, b). Positive control regions included the ventral juxtaventromedial LHA (LHAjvv; Fig. 3i) for CaMKIla [16], the more caudal juxtadorsomedial LHA (LHAjd; Fig. 3k) for orexin [17], the anterior hypothalamic nucleus (AHN; Fig. 4m) for $\mathrm{MCH}$ [17], and the paraventricular hypothalamus ( $\mathrm{PVH}$; Fig. 3o) for galanin [18].

We previously reported $87 \%$ of parvalbumin-positive $\left(\mathrm{PV}^{+}\right)$ LHAad neurons coexpress WFA, while $62 \%$ of WFA positive (WFA ${ }^{+}$) neurons coexpress PV. However, $\mathrm{PV}^{+} / \mathrm{PNN}$ glutamate neurons have been reported in the ventral lateral hypothalamus [19]. We determined whether WFA ${ }^{+}$neurons of the LHAad primarily colocalize with GABAergic or glutamatergic markers.

GAD65/67 expression did not colocalize with WFA in the LHAad (Fig. 3d). However, this marker often measures synaptic terminals [20]; it could not be determined if expression was on the terminals or cell body. Staining for GABA labels cell bodies [21] which we found to co-localize with WFA in the LHAad (Fig. 3e). Excitatory terminals are particularly enriched on $\mathrm{PV}^{+} \mathrm{PNN}$-surrounded cells [22], and VGLUT2 is the excitatory synaptic marker that predominates in the anterior LHA $[23,24]$. We found that colocalization of VGLUT2 with WFA was robust (Fig. 3f). Similar to GAD65/67, it could not be determined if VGLUT2 was expressed on the terminals or the cell body. Staining for glutamate also labels cell bodies [19]. Glutamate did not co-localize in the majority of cells with WFA except for the most ventral tier of the LHAad (Fig. 3g). CamKIlla did not colocalize with WFA in the LHAad (Fig. $3 \mathrm{~h}$ ) but was expressed in the LHAjvv positive control region (Fig. 3i).

Orexin, $\mathrm{MCH}$, and galanin neuropeptides are separate, nonoverlapping, anatomically adjacent populations of neurons that regulate both food seeking and reinstatement of cocaine seeking $[10,25-27]$. Therefore, we determined whether PNN/ECMsurrounded neurons of the LHAad expressed orexin, $M C H$, or galanin. Orexin did not colocalize with WFA in the LHAad (Fig. 3j) but was expressed in the LHAjd positive control region (Fig. $3 \mathrm{k}$ ). $\mathrm{MCH}$ did not co-localize with WFA in the LHAad (Fig. 3l) but was expressed in the AHN positive control region (Fig. $3 \mathrm{~m}$ ). Galanin was expressed at very low levels in the LHAad (Fig. 3n) but was expressed in the PVH positive control region (Fig. 3o). In summary, our findings show that the neurons surrounded by PNNs/ECM in the LHAad are primarily GABAergic $\mathrm{PV}^{+}$neurons that receive dense glutamatergic input and do not colocalize with feedingrelated peptides of the hypothalamus.

LHAad is embedded within the neurocircuitry of motivated behavior The neurocircuitry of the PNN/ECM-rich LHAad was characterized within the mesocorticolimbic system. We have recently found that PNNs in the prelimbic PFC may regulate pyramidal neuron output
[6], which is known to drive reinstatement [2]. To determine if the prelimbic PFC provides input into the LHAad (PL to $L H)$, retrobeads were unilaterally injected into the LHAad (Fig. 4a), and we examined retrobead expression in the prelimbic PFC (Fig. 4b). There was robust retrobead expression in the prelimbic PFC (Fig. 4c, j; 397.7 $\pm 37.28 \mathrm{RB}^{+}$cells $/ \mathrm{mm}^{2} ; n=4$ ) indicating that the prelimbic PFC provides heavy input into the LHAad. To determine whether the LHAad projects to the VTA (LH to VTA), retrobeads were unilaterally injected into the VTA (Fig. 4d), and we examined retrobead expression in the LHAad. Retrobead injections into the VTA resulted in moderate (Fig. 4e, j; $210.4 \pm 15.23 \mathrm{RB}^{+}$cells $/ \mathrm{mm}^{2}$; $n=6$ ) retrograde expression in the LHAad. To determine if the LHAad projects to the prelimbic PFC (LH to PL), retrobeads were unilaterally injected into the prelimbic PFC (Fig. 4f), and we examined retrobead expression in the LHAad. Retrobead injections into the prelimbic PFC resulted in moderate (Fig. $4 \mathrm{~g}$, j; $221.2 \pm 14.25 \mathrm{RB}^{+}$cells $/ \mathrm{mm}^{2} ; n=3$ ) retrobead expression in the LHAad. To determine if the LHAad projects to the nucleus accumbens (LH to NA), retrobeads were unilaterally injected into the nucleus accumbens (Fig. 4h), and we examined retrobead expression in the LHAad. The majority of retrobead injections into the nucleus accumbens spanned the shell, core, and the border region between, which has been referred to as the "shore" [28, 29]. Retrobead injections into the nucleus accumbens resulted in relatively minimal (Fig. 4i, j; $26.55 \pm 5.079 \mathrm{RB}^{+}$cells $/ \mathrm{mm}^{2} ; n=4$ ) retrobead expression in the LHAad. Three RB rats were omitted from the analysis for placement outside the area of interest ( $\mathrm{LH}$ to VTA: $n=2$; PL to LH: $n=1$ ) and excluded from analysis. In addition to number of cells (Fig. 4j), the total area of each PL to LH RB ${ }^{+}$cell $(27.00 \pm 1.132$ pixels) was greater than that of LH to VTA (15.09 \pm 0.65 pixels), $\mathrm{LH}$ to $\mathrm{PL}(14.12 \pm 1.275$ pixels), and LH to NA (10.50 \pm 0.8341 pixels) $\mathrm{RB}^{+}$cells (data not shown). In summary, the LHAad is centrally embedded within the circuitry of motivated behavior (Fig. 4k) via robust prelimbic PFC inputs and moderate outputs to the VTA and the prelimbic PFC.

\section{DISCUSSION}

We recently described a novel and remarkably discrete region, the LHAad, that is demarcated by dense PNN/ECM expression and that is required for the acquisition of cocaine CPP and SA. Here we show that the LHAad PNN/ECM is required for cocaine but not sucrose cue-induced reinstatement in SA rats. The LHAad appears to be a unique region of the LHA in that it is devoid or expresses only very low levels of feeding-related neuropeptides and mediates cue-induced reinstatement to cocaine but not to sucrose.

One caveat to our findings is that intra-LHAad Ch-ABC administration led to a reduction of inactive lever pressing during cue-induced reinstatement, suggesting possible general effects on locomotor behavior. However, this effect was not observed during sucrose-induced reinstatement. Moreover, our previous study examining cocaine-induced CPP did not find differences in locomotor activity between vehicle and $\mathrm{Ch}$-ABC-treated rats. Overall, these data suggest that degradation of PNN/ECM in the LHAad blocks cocaine cue-induced reinstatement.

\section{Drugs of abuse and the PNN/ECM}

PNN/ECM expression in specific brain regions appear necessary for drug-induced behavior $[6,7]$ and drug exposure can alter the PNN/ECM [4, 30, 31]. Matrix metalloproteinases (MMPs) are endogenous enzymes that degrade the PNN/ECM. Paradoxically, both increases in MMP activity and expression of PNN/ECM components have been reported following drug exposure [4, 30, 32]. Decreases in PNN/ECM expression have been found during abstinence or extinction from heroin self-administration, while increases were found just after cue-induced reinstatement [4]. The changes in PNN/ECM expression following drugs of abuse likely 
depends on the brain region and the phase of the behavioral paradigm.

\section{The LHA PNN/ECM}

High PNN/ECM expression within the neurocircuitry of motivated behavior was hypothesized to provide insight into novel circuitry driving addiction. The LHA is an essential interface between motivational action and goal-oriented behavior [33], and reported to express high levels of PNN/ECM [34]. This led us to discover the more discrete LHAad and its role in cocaine seeking. Regarding the mechanism by which the PNN/ECM-rich LHAad might mediate cocaine seeking, components within the PNN may regulate activation of their underlying neurons. For example, very recent work demonstrated that experience alters the activity of PV cells in the hippocampus through the PNN component brevican [35], which mediates changes through altered AMPA receptors and voltage-gated potassium channels critical for fast-spiking of PV cells. Furthermore, the discrete, high-level expression of PNNs/ ECM that is observed in the LHAad may be due to a lack of link protein [36] with heavy levels of aggrecan [37].

\section{LHAad neuron phenotype}

PNN/ECM-surrounded neurons are not always $\mathrm{PV}^{+}$GABAergic interneurons. In the mouse anterior hypothalamus they are positive for calretinin and enkephalin but not for PV [38]. In the ventral LHA PV ${ }^{+}$glutamate neurons co-localize with WFA [19]. In the LHAad we find that WFA colabels with PV, VGLUT2, and GABA but not GAD65/67 or glutamate. These findings indicate the PNN/ECM-rich LHAad receives dense glutamatergic input and is composed predominantly of GABAergic, PV neurons. VGLUT2 is the excitatory synaptic marker that predominates in the anterior LHA $[23,24]$. Candidate sources of glutamate input into the LHAad are the prelimbic PFC and basolateral amygdala (BLA) [23].

We replicate previous studies reporting no discernable levels of $\mathrm{MCH}$ or orexin/hypocretin [17] and report no detectable levels of galanin in the LHAad. The lack of feeding neuropeptides on PNNsurround GABAergic neurons in the LHAad may explain why removal of $\mathrm{PNNs} / \mathrm{ECM}$ with $\mathrm{Ch}-\mathrm{ABC}$ blocked cocaine-seeking but not sucrose-seeking behavior. However, we did observe a weak trend for a reduction in the number of active lever presses during cue-induced reinstatement for sucrose, suggesting that LHAad PNNs/ECM may partially contribute to the rewarding effects of highly palatable food such as sucrose. The hedonic impact of the reward stimulus, cocaine versus sucrose, may account for significant findings in cocaine but not sucrose self-administering animals.

\section{LHAad neurocircuitry}

It is well established that the mesocorticolimbic system regulates reinstatement of drug seeking. This includes the VTA (meso), PFC (cortico), and NA (limbic). Interconnectivity between these regions and the LHAad was assessed using retrobeads. We report robust connectivity from the PL to the LHAad, moderate connectivity from the LHAad to both the PL and the VTA, and minimal connectivity from the LHAad to the NA. One caveat with this approach is that these connections may involve PNN/ECM surrounded neurons or other neurons of the LHAad, which may be projection neurons or resident interneurons. Retrobeads are not transported trans-synaptically [13], suggesting these are monosynaptic connections between the PNN/ECM-dense region of the LHAad with the PL and VTA. PNN/ECM surrounded LHAad neurons primarily co-localize with $P V$, which is known for classifying fast-spiking GABAergic interneurons $[39,40]$. However, PV projection neurons have also been found [19,41,42], including neurons within the LHA [19]. Our retrobead findings suggest that the LHAad PNN/ECM is interconnected within the mesocortical system.
In conclusion, we describe a novel area of the LHA containing dense PNN/ECM-surrounded neurons in the LHAad that are primarily GABAergic $\mathrm{PV}^{+}$neurons, receive heavy glutamatergic (VGLUT2) input, but do not colocalize with the feeding-related neuropeptides of the hypothalamus. The PNN/ECM-rich LHAad is centrally positioned within the neurocircuitry of motivated behavior and may be acting as an integration site for cocainemediated rewards. Future studies should identify in greater detail the phenotype of these projections and the impact of LHAad PNN/ ECM degradation on synaptic signaling within this circuit.

\section{ACKNOWLEDGEMENTS}

The authors wish to thank Dr. Daniel S. Zahm (Saint Louis University School of Medicine) for helpful discussions and Dr. John Harkness (Washington State University Vancouver) for development of PIPSQUEAK software for anatomical quantification of the retrobead study.

\section{FUNDING AND DISCLOSURE}

This work was funded by National Institute of Health (NIH) DA033404 and DA040965 to BAS and Washington State Initiative 171, Washington State University Alcohol and Drug Abuse Research Program (ADARP), 124777 and NIH DA045082 to JMB.

\section{ADDITIONAL INFORMATION}

Supplementary Information accompanies this paper at (https://doi.org/10.1038/ s41386-018-0212-8)

Competing interests: The authors declare no competing interests.

Publisher's note: Springer Nature remains neutral with regard to jurisdictional claims in published maps and institutional affiliations.

\section{REFERENCES}

1. O'Brien C. Addiction and dependence in DSM-V. Addiction. 2011;106:866-7.

2. Kalivas PW, Volkow ND. The neural basis of addiction: a pathology of motivation and choice. Am J Psychiatry. 2005;162:1403-13.

3. Mash DC, ffrench-Mullen J, Adi N, Qin Y, Buck A, Pablo J. Gene expression in human hippocampus from cocaine abusers identifies genes which regulate extracellular matrix remodeling. PLoS ONE. 2007;2:e1187.

4. Van den Oever MC, Lubbers BR, Goriounova NA, Li KW, Van der Schors RC, Loos $M$, et al. Extracellular matrix plasticity and GABAergic inhibition of prefrontal cortex pyramidal cells facilitates relapse to heroin seeking. Neuropsychopharmacology. 2011;35:2120-33.

5. Blacktop JM, Todd RP, Sorg BA. Role of perineuronal nets in the anterior dorsal lateral hypothalamic area in the acquisition of cocaine-induced conditioned place preference and self-administration. Neuropharmacology. 2017;118:124-36.

6. Slaker M, Churchill L, Todd RP, Blacktop JM, Zuloaga DG, Raber J, et al. Removal of perineuronal nets in the medial prefrontal cortex impairs the consolidation and reconsolidation of cocaine-induced conditioned place preference. J Neurosci. 2015;35:4190-202.

7. Xue YX, Xue LF, Liu JF, He J, Deng JH, Sun SC, et al. Depletion of perineuronal nets in the amygdala to enhance the erasure of drug memories. J Neurosci. 2014;34:6647-58

8. Nieuwenhuys R, Geeraedts LM, Veening JG. The medial forebrain bundle of the rat. I. General introduction. J Comp Neurol. 1982;206:49-81.

9. Olds J, Milner P. Positive reinforcement produced by electrical stimulation of septal area and other regions of rat brain. J Comp Physiol Psychol. 1954:47:419-27.

10. Berthoud HR, Munzberg $\mathrm{H}$. The lateral hypothalamus as integrator of metabolic and environmental needs: from electrical self-stimulation to opto-genetics. Physiol Behav. 2011;104:29-39.

11. Marchant NJ, Rabei R, Kaganovsky K, Caprioli D, Bossert JM, Bonci A, et al. A critical role of lateral hypothalamus in context-induced relapse to alcohol seeking after punishment-imposed abstinence. J Neurosci. 2014:34:7447-57.

12. Bruckner G, Bringmann A, Hartig W, Koppe G, Delpech B, Brauer K. Acute and long-lasting changes in extracellular-matrix chondroitin-sulphate proteoglycans induced by injection of chondroitinase $A B C$ in the adult rat brain. Exp Brain Res. 1998;121:300-10.

13. Schofield BR. Retrograde axonal tracing with fluorescent markers. Curr Protoc Neurosci. 2008;43:1-17. 
14. Hartig W, Brauer K, Bruckner G. Wisteria floribunda agglutinin-labelled nets surround parvalbumin-containing neurons. Neuroreport. 1992;3:869-72.

15. Slaker ML, Harkness JH, Sorg BA. A standardized and automated method of perineuronal net analysis using Wisteria floribunda agglutinin staining intensity. IBRO Rep. 2016;1:54-60.

16. Ouimet CC, McGuinness TL, Greengard P. Immunocytochemical localization of calcium/calmodulin-dependent protein kinase II in rat brain. Proc Natl Acad Sci USA. 1984;81:5604-8.

17. Hahn JD. Comparison of melanin-concentrating hormone and hypocretin/orexin peptide expression patterns in a current parceling scheme of the lateral hypothalamic zone. Neurosci Lett. 2010;468:12-17.

18. Leibowitz SF, Akabayashi A, Wang J. Obesity on a high-fat diet: role of hypothalamic galanin in neurons of the anterior paraventricular nucleus projecting to the median eminence. J Neurosci. 1998;18:2709-19.

19. Meszar Z, Girard F, Saper CB, Celio MR. The lateral hypothalamic parvalbuminimmunoreactive (PV1) nucleus in rodents. J Comp Neurol. 2012;520:798-815.

20. Esclapez M, Tillakaratne NJ, Kaufman DL, Tobin AJ, Houser CR. Comparative localization of two forms of glutamic acid decarboxylase and their mRNAs in rat brain supports the concept of functional differences between the forms. J Neurosci. 1994;14:1834-55.

21. Ye L, Allen WE, Thompson KR, Tian Q, Hsueh B, Ramakrishnan C, et al. Wiring and molecular features of prefrontal ensembles representing distinct experiences. Cell. 2016;165:1776-88.

22. Carulli D, Foscarin S, Faralli A, Pajaj E, Rossi F. Modulation of semaphorin $3 A$ in perineuronal nets during structural plasticity in the adult cerebellum. Mol Cell Neurosci. 2013;57:10-22.

23. Yeoh JW, James MH, Jobling P, Bains JS, Graham BA, Dayas CV. Cocaine potentiates excitatory drive in the perifornical/lateral hypothalamus. J Physiol. 2012;590:3677-89.

24. Ziegler DR, Cullinan WE, Herman JP. Distribution of vesicular glutamate transporter mRNA in rat hypothalamus. J Comp Neurol. 2002;448:217-29.

25. Aston-Jones G, Smith RJ, Moorman DE, Richardson KA. Role of lateral hypothalamic orexin neurons in reward processing and addiction. Neuropharmacology. 2009;56:112-21.

26. Ogbonmwan YE, Sciolino NR, Groves-Chapman JL, Freeman KG, Schroeder JP, Edwards GL, et al. The galanin receptor agonist, galnon, attenuates cocaineinduced reinstatement and dopamine overflow in the frontal cortex. Addict Biol. 2015;20:701-13.

27. Chung S, Hopf FW, Nagasaki H, Li CY, Belluzzi JD, Bonci A, et al. The melaninconcentrating hormone system modulates cocaine reward. Proc Natl Acad Sci USA. 2009;106:6772-7.

28. Prinssen EP, Balestra W, Bemelmans FF, Cools AR. Evidence for a role of the shell of the nucleus accumbens in oral behavior of freely moving rats. J Neurosci. 1994;14:1555-62.
29. Rebec GV, Christensen JR, Guerra C, Bardo MT. Regional and temporal differences in real-time dopamine efflux in the nucleus accumbens during free-choice novelty. Brain Res. 1997;776:61-67.

30. Smith AC, Kupchik YM, Scofield MD, Gipson CD, Wiggins A, Thomas CA, et al. Synaptic plasticity mediating cocaine relapse requires matrix metalloproteinases. Nat Neurosci. 2014;17:1655-7.

31. Vazquez-Sanroman D, Leto K, Cerezo-Garcia M, Carbo-Gas M, Sanchis-Segura C, Carulli D et al. (2015). The cerebellum on cocaine: plasticity and metaplasticity. Addict Biol. 20;(5): 941-55

32. Brown TE, Forquer MR, Cocking DL, Jansen HT, Harding JW, Sorg BA. Role of matrix metalloproteinases in the acquisition and reconsolidation of cocaineinduced conditioned place preference. Learn Mem. 2007;14:214-23.

33. Mogenson GJ, Jones DL, Yim CY. From motivation to action: functional interface between the limbic system and the motor system. Prog Neurobiol. 1980;14:69-97.

34. Seeger G, Brauer K, Hartig W, Bruckner G. Mapping of perineuronal nets in the rat brain stained by colloidal iron hydroxide histochemistry and lectin cytochemistry. Neuroscience. 1994;58:371-88.

35. Favuzzi E, Marques-Smith A, Deogracias R, Winterflood CM, Sanchez-Aguilera A, Mantoan $L$, et al. Activity-dependent gating of parvalbumin interneuron function by the perineuronal net protein brevican. Neuron. 2017;95:639-55:e610.

36. Horii-Hayashi N, Sasagawa T, Nishi M. Insights from extracellular matrix studies in the hypothalamus: structural variations of perineuronal nets and discovering a new perifornical area of the anterior hypothalamus. Anat Sci Int. 2017;92:18-24.

37. Dauth S, Grevesse T, Pantazopoulos H, Campbell PH, Maoz BM, Berretta S, et al. Extracellular matrix protein expression is brain region dependent. J Comp Neurol. 2016;524:1309-36

38. Horii-Hayashi N, Sasagawa T, Hashimoto T, Kaneko T, Takeuchi K, Nishi M (2015). A newly identified mouse hypothalamic area having bidirectional neural connections with the lateral septum: the perifornical area of the anterior hypothalamus enriched in chondroitin sulfate proteoglycans. Eur J Neurosci.42; (6):2322-2334.

39. Kawaguchi Y. Physiological subgroups of nonpyramidal cells with specific morphological characteristics in layer II/III of rat frontal cortex. J Neurosci. 1995; 15:2638-55.

40. Cowan RL, Wilson CJ, Emson PC, Heizmann CW. Parvalbumin-containing GABAergic interneurons in the rat neostriatum. J Comp Neurol. 1990;302:197-205

41. Freund TF. GABAergic septohippocampal neurons contain parvalbumin. Brain Res. 1989;478:375-81.

42. Lee AT, Vogt D, Rubenstein JL, Sohal VS. A class of GABAergic neurons in the prefrontal cortex sends long-range projections to the nucleus accumbens and elicits acute avoidance behavior. J Neurosci. 2014;34:11519-25. 